BNL-113257-2016-JA

\title{
Two-Dimensional Layered Oxide Structures Tailored by Self Assembled Layer Stacking via Interfacial Strain
}

\author{
Wenrui Zhang, Aiping Chen, Mingtao Li, Leigang Li, \\ Yuanyuan Zhu, Zhenhai Xia, Ping Lu, Philippe Boullay, \\ Lijun Wu, Yimei Zhu, Judith L. MacManus-Driscoll, Quanxi Jia, \\ Honghui Zhou, Jagdish Narayan, Xinghang Zhang, Haiyan Wang
}

Submitted to ACS Applied Materials \& Interfaces

July 2016

\begin{abstract}
Condensed Matter Physics and Material Science Department
Brookhaven National Laboratory
\end{abstract}

\section{U.S. Department of Energy USDOE Office of Science, Basic Energy Sciences}




\section{DISCLAIMER}

This report was prepared as an account of work sponsored by an agency of the United States Government. Neither the United States Government nor any agency thereof, nor any of their employees, nor any of their contractors, subcontractors, or their employees, makes any warranty, express or implied, or assumes any legal liability or responsibility for the accuracy, completeness, or any third party's use or the results of such use of any information, apparatus, product, or process disclosed, or represents that its use would not infringe privately owned rights. Reference herein to any specific commercial product, process, or service by trade name, trademark, manufacturer, or otherwise, does not necessarily constitute or imply its endorsement, recommendation, or favoring by the United States Government or any agency thereof or its contractors or subcontractors. The views and opinions of authors expressed herein do not necessarily state or reflect those of the United States Government or any agency thereof. 


\title{
Two-Dimensional Layered Oxide Structures Tailored by Self- Assembled Layer Stacking via Interfacial Strain
}

\author{
Wenrui Zhang ${ }^{1 \dagger}$, Aiping Chen ${ }^{2 \dagger}$, Mingtao $\mathrm{Li}^{3,4 \dagger}{ }^{4}$, Leigang $\mathrm{Li}^{1}$, Yuanyuan Zhu ${ }^{1}$, Zhenhai Xia ${ }^{3}$, Ping Lu ${ }^{5}$, \\ Philippe Boullay ${ }^{6}$, Lijun Wu ${ }^{7}$, Yimei Zhu ${ }^{7}$, Judith L. MacManus-Driscoll ${ }^{8}$, Quanxi, Jia ${ }^{9}$, Honghui \\ Zhou ${ }^{10}$, Jagdish Narayan ${ }^{10}$, Xinghang Zhang ${ }^{1,11}$, Haiyan Wang ${ }^{1,2 \star}$ \\ ${ }^{1}$ Department of Materials Science and Engineering, Texas A\&M University, 3128 TAMU, College Station, Texas 77843, USA, \\ ${ }^{2}$ Department of Electrical and Computer Engineering, Texas A\&M University, 3128 TAMU, College Station, Texas 77843, USA, \\ ${ }^{3}$ Department of Materials Science and Engineering, and Department of Chemistry, University of North Texas, Denton, Texas 76203, \\ USA, \\ ${ }^{4}$ International Research Center for Renewable Energy, State key Laboratory of Multiphase Flow in Power Engineering, Xi'an \\ Jiaotong University, Xi'an, Shaanxi 710049, P. R. China, \\ ${ }^{5}$ Sandia National Laboratories, Albuquerque, New Mexico 87185, USA \\ ${ }^{6}$ CRISMAT, CNRS UMR 6508, ENSICAEN, 6 Boulevard Maréchal Juin, F-14050 Caen Cedex 4, France, \\ ${ }^{7}$ Condensed Matter Physics \& Materials Science Department, Brookhaven National Laboratory, Upton, New York 11973, USA, \\ ${ }^{8}$ Department of Materials Science, University of Cambridge, Cambridge, CB2 3QZ, UK, \\ ${ }^{9}$ Center for Integrated Nanotechnologies, MS K771, Los Alamos National Laboratory, Los Alamos, New Mexico 87545, USA, \\ ${ }^{10}$ Department of Materials Science and Engineering, NSF Center for Advanced Materials and Smart Structures, North Carolina State \\ University, Raleigh, NC 27695, USA \\ ${ }^{11}$ Department of Mechanical Engineering, Texas A\&M University, 3128 TAMU, College Station, Texas 77843, USA.
}

${ }^{\star}$ E-mail: wangh@ece.tamu.edu

${ }^{\dagger}$ These authors contributed equally to this work 
Two-dimensional (2D) nanostructures emerge as one of leading topics in fundamental materials science and could enable next generation nanoelectronic devices ${ }^{1-13}$. Beyond graphene and molybdenum disulphide, layered complex oxides are another large group of promising $2 \mathrm{D}$ candidates because of their strong interplay of intrinsic charge, spin, orbital and lattice ${ }^{4,5}$. As a fundamental basis of heteroepitaxial thin film growth, interfacial strain can be used to design materials exhibiting new phenomena beyond their conventional form ${ }^{6}$. Here we report the strain-driven self-assembly of Bismuth-based supercells (SC) with a 2D layered structure, and elucidate the fundamental growth mechanism with combined experimental tools and first-principles calculations. The study revealed that the new layered structures were formed by the strain-enabled self-assembled atomic layer stacking, i.e., alternative growth of $\mathrm{Bi}_{2} \mathrm{O}_{2}$ layer and $\left[\mathrm{Fe}_{0.5} \mathrm{Mn}_{0.5}\right] \mathrm{O}_{6}$ layer. The strain-driven approach is further demonstrated in other SC candidate systems with promising room-temperature multiferroic properties. This well-integrated theoretical and experimental study inspired by the Materials Genome Initiatives opens up a new avenue in searching and designing novel 2D layered complex oxides with enormous promises. 
Tremendous fundamental and practical interests in two-dimensional (2D) layered functional materials, rekindled by the discovery of the graphene nanosheets ${ }^{7,8}$, is based on their exotic electronic properties ${ }^{9-11}$ and the fascinating practical applications in sensing ${ }^{12}$, and energy aspects ${ }^{13}$. Layered compounds are an intriguing material family with diverse material systems, which can be categorized as non-oxide based ones including graphene ${ }^{7}$, transition metal dichalcogenides ${ }^{11,14}$, boron nitride ${ }^{15}, \mathrm{X}_{2} \mathrm{Te}_{3}$ $(\mathrm{X}=\mathrm{Bi}, \mathrm{Sb}){ }^{16}, \mathrm{AB}_{2}(\mathrm{~A}=\mathrm{Mo}, \mathrm{Ta}, \mathrm{Nb}, \mathrm{Ni}, \mathrm{W}, \mathrm{B}=\mathrm{S}, \mathrm{Se}, \mathrm{Te}){ }^{17}$, and oxide-based transition metal compounds ${ }^{18-20}$. Non-oxide based 2D layered materials have attracted wide attention in the past few years with most efforts devoted to synthesizing large scale layered materials ${ }^{17,21}$, understanding their basic physical properties ${ }^{22,23}$, and enabling new generation electronic devices ${ }^{24}$. Nevertheless, the research scope of layered functional compounds is far beyond the above summary. Complex oxides themselves, with the discovery of high temperature superconductivity ${ }^{25,26}$, colossal magnetoresistance ${ }^{27}$, and single-phase multiferroics ${ }^{28,29}$, have been the focus of condensed matters physics and material science for decades. Tuning the functionalities by manipulating the interplay among charge, spin, orbital and lattice through the multilayers, superlattices and vertical architectures has achieved remarkable success ${ }^{30,31}$.

Recently developed advanced thin film growth technologies and state-of-the-art characterization techniques also provide unprecedented opportunities to explore, understand and utilize complex oxides in 2D layered form with new functionalities ${ }^{32}$. For example, commensurate or incommensurate modulated layered structures show giant anisotropy of physical properties, such as magnetoresistance ${ }^{33}$, electronic/ionic conductivity ${ }^{34}$ and thermoelectric properties ${ }^{35}$. In addition, perovskite-related layered 
materials like Aurivillius and Ruddlesden-Popper phases exhibit a great variability with respect to metal cation substitution because of their unique ionic structural framework ${ }^{36}$. To meet the demands of new materials for future functional devices and consider the enormously large selections of complex oxides, seeking new layered oxide compounds with desired multifunctionalities holds great promises and deserves a long term research effort. One method to growth such a structure is to artificially control the stacking sequence of selected 2D atomic crystal planes based on the molecular beam epitaxy technique ${ }^{18}$. In parallel with the MBE method which artificially controls the stacking process, self-assembly of layer stacking is another promising route for designing new layered structures. Introducing the biaxial strain through the substrate in thin film epitaxy growth has served as a very effective way to manipulate the film microstructure and physical properties ${ }^{6}$. Inspired by the Materials Genome Initiatives, here we demonstrate a strain-driven self-assembled method to grow a new family of 2D layered oxide structures and uncover their fundamental growth mechanism based on a well-integrated theoretical and experimental approach. The results could open up a new avenue in designing novel 2D layered oxide structures with enormous possibilities for new functionalities and devices.

The $\mathrm{Bi}_{3} \mathrm{Fe}_{2} \mathrm{Mn}_{2} \mathrm{O}_{10+\delta}$ (BFMO) SC films were grown on $\mathrm{LaAlO}_{3}$ (LAO) (001) substrates using pulsed laser deposition from a stoichiometric $\mathrm{Bi}_{2} \mathrm{FeMnO}_{6}$ target (see Supplementary section I for experimental details). Aberration-corrected scanning transmission electron microscope (STEM) in high angle annular dark-field (HAADF) mode was conducted on the as-prepared BFMO films along the substrate [100] zone axis, and a representative image is shown in Fig. 1a. An interlayer structure $(\sim 5 \mathrm{~nm})$ with a 
higher degree of tetragonality $(\mathrm{c} / \mathrm{a}=1.15)$ is formed between the underlying substrate and the deposited film. Biaxial elastic strain is found to build up in this interlayer. Beyond it, a clear new layered phase can be identified accompanied with significant strain relaxation by a modified geometric phase analysis ${ }^{37}$. Based on the STEM image, cation positions of the new phase have been determined as shown in the atomic model in our previous report ${ }^{29}$. However it lacks the detailed information on the oxygen lattice sites and their interaction with cations, which limits the understanding of the basic crystal structure of this new phase as well as the growth mechanism.

For this purpose, a STEM annular bright field (ABF) imaging method was employed, which has demonstrated its powerful capability to image light elements such as oxygen, nitrogen, lithium and even hydrogen ${ }^{38}$. Fig. $1 \mathrm{~b}$ shows the high-resolution STEM ABF images of BFMO SC along the $[100]_{\mathrm{p}}$ zone axis ( $P$ stands for the 'pseudocubic' phase as the simplified pseudo-cubic phase is applied in this work to discuss the zone axis in SC). These images were slightly processed by low-pass filter to improve signal/noise ratio. A periodic arrangement of the SC structure is marked with yellow rectangles. Fig. 1c shows an enlarged image of $\mathrm{Bi}_{2} \mathrm{O}_{2}$ layer and $\left[\mathrm{Fe}_{0.5} \mathrm{Mn}_{0.5}\right] \mathrm{O}_{6}$ layer with atomic intensity profiles for probing oxygen ion positions. The contrast intensity of the oxygen columns between Bi bilayers varies from place to place, indicating stoichiometric oxygen atomic columns (filled circles) and ones containing oxygen vacancies (voided circles) in $\mathrm{Bi}_{2} \mathrm{O}_{2}$ layers. Slight oxygen deficiency has also been observed in $\left[\mathrm{Fe}_{0.5} \mathrm{Mn}_{0.5}\right] \mathrm{O}_{6}$ octahedra layers due to mixed valences of $\mathrm{Fe} / \mathrm{Mn}$ ions.

The enlarged images of BFMO SC films along $[100]_{p}$ and $[010]_{p}$ directions are shown in Fig. 1e and 1f, respectively. It is interesting to note that the $\mathrm{Bi}_{2} \mathrm{O}_{2}$ sheets exhibit 
an incommensurate stacking having a 1:1 matching with $\left[\mathrm{Fe}_{0.5} \mathrm{Mn}_{0.5}\right] \mathrm{O}_{6}$ octahedra in the $[100]_{\mathrm{p}}$ zone axis however a $4: 3$ matching in the $[010]_{\mathrm{p}}$ axis. Fig. $1 \mathrm{~d}$ and g present the atomic model with referred oxygen positions based on the above ABF imaging results. Most of the oxygen positions have been determined and marked in yellow. Those with light blue and orange colored ones stay too close to the heavier cations to be identified precisely. Therefore first-principles calculations were conducted to resolve the undetermined oxygen positions based on the considerations of lattice formation energy.

The oxygen positions are calculated through an unbiased search for the global minimum (GM) structures of $\mathrm{Bi}_{6} \mathrm{Fe}_{4} \mathrm{Mn}_{4} \mathrm{O}_{21+\delta}$ by employing CALYPSO code with cation constraints ${ }^{39}$. A primitive cell containing $6 \mathrm{Bi}, 4 \mathrm{Fe}$ and $4 \mathrm{Mn}$ ions is selected as the cation frame for reasonable calculation cost. Fig. 1h shows the derived structure of $\mathrm{Bi}_{6} \mathrm{Fe}_{4} \mathrm{Mn}_{4} \mathrm{O}_{22}$ based on the reduced primitive cell according to the ideal film stoichiometry. The $\left[\mathrm{Fe}_{0.5} \mathrm{Mn}_{0.5}\right] \mathrm{O}_{6}$ octahedra are contacted in edge-share mode in direction $[100]_{\mathrm{p}}$ and in vertex-share mode in direction $[010]_{\mathrm{p}}$. After the locations of all the atomic positions were determined, full geometry relaxation was carried out employing PBE+U with the effective Hubbard interaction $U_{\text {eff }} 4.6 / 4.0 \mathrm{eV}$ for Fe/Mn. The detailed structural information including oxygen positions is provided in Supplementary Table S1.

To fully investigate the SC crystal structure, the detailed Fe/Mn distribution inside is needed. Atomic-scale energy-dispersive X-ray spectroscopy (EDS) mapping was thus carried out to obtain the Fe/Mn elemental distribution of the BFMO SC structure. Fig. 2a and $2 \mathrm{f}$ show the high-resolution HAADF STEM image of the SC structure along the $[100]_{p}$ and $[010]_{p}$ zone axis used for EDS mapping. The $\mathrm{Bi}_{2} \mathrm{O}_{2}$ stacking is marked out 
with triangles, and the Fe/Mn atomic position is located within the oxygen octahedron. Fe and Mn ions cannot be clearly distinguished from each other in the STEM image due to their close electron scattering powers and ionic radius. Fig. 2(b-d) and 2(g-i) show the EDS maps of $\mathrm{Bi}(\mathrm{L}+\mathrm{M})$, $\mathrm{Mn}\left(\mathrm{K}_{\alpha}\right)$ and $\mathrm{Fe}\left(\mathrm{K}_{\alpha}\right)$ along two axes of $[100]_{\mathrm{p}}$ and $[010]_{\mathrm{p}}$, respectively. The lattice averaging method is used to improve the signal/noise ratio significantly, which allows direct visualization of atomic columns of different elements in the projection in the EDS mode ${ }^{40}$. Fe and $\mathrm{Mn}$ ions have been found to occupy the identical lattice sites in the SC structure, and Bi ions have the same arrangement as observed in the STEM images. Fig. 4e and 4j show their composite color maps of the Bi ( $\mathrm{L}+\mathrm{M}$, in red), $\mathrm{Mn}\left(\mathrm{K}_{\alpha}\right.$, in green) and Fe ( $\mathrm{K}_{\alpha}$, in blue) along the two directions. Based on these experimental observations, Bi ions present a clear periodic arrangement without any intermixing with others, while Fe and $\mathrm{Mn}$ ions are suggested to be randomly distributed in their lattice sites without any preferential ordering identified.

The overall crystal symmetry and structure of the BFMO SC has been further explored by precession electron diffraction (PED), which has been proven to be a valuable technique for the structure determination of unknown phases, even in the form of thin films ${ }^{41,42}$. Sections through the reciprocal space can be used for a first visual search of systematic absences (Fig. 3a and 3b), allowing us to propose an A-centering of the cell. The LAO (001) substrate was used as an internal reference and the obtained values can be considered as a good starting point for structure solution. Considering this centering and the lattice mismatch between the BFMO SC phase and the LAO substrate, the peak picking operation performed during the intensity integration procedure allows eliminating the most intense reflections coming from the substrate (Supplementary Fig. 
S1). The data set yielded data completeness over $80 \%$ (total number of reflections in the $0.9^{\circ} \mathrm{A}$ resolution shell), sufficient for the structure solution process. The structure solution was performed using the program Superflip ${ }^{43}$ assuming kinematical diffraction intensities. The obtained structural model was refined using the charge flipping procedure and completed using Fourier difference maps leading to an orthorhombic unit cell with the space group of Amm2 (see Supplementary Fig. S2 and Table S2 for details).

Fig. 3c describes the schematic crystal structures used in first-principles calculations and PED results. Their structural parameters are listed in Fig. 3d. The orthorhombic unit cell obtained from PED data agrees well with the monoclinic phase in theoretical calculations in terms of lattice parameters, except that different basic units of the SC phase are selected to represent the entire structure. Here the crystal structure for first-principles calculations is selected from the point view of layered stacking growth, which agrees with the model used in previous oxygen lattice sites calculation (Supplementary Fig. S3). The symmetry analysis of the PED data indicates the space group of Amm2 as the most probable symmetry of the BFMO SC structure, which is slightly higher than $\mathrm{P} 2{ }_{1} / \mathrm{m}$ as a monoclinic phase. The Amm2 symmetry neglects a small $\left[\mathrm{Fe}_{0.5} \mathrm{Mn}_{0.5}\right] \mathrm{O}_{6}$ octahedra distortion by approximation and is used to describe this $\mathrm{SC}$ structure as a 'pseudo-orthorhombic' phase.

Based on the above structural results, the growth mechanism of BFMO SC structure is finally proposed in consideration of epitaxial strain relaxation and interfacial reconstructions, and confirmed with experimental demonstrations. For the initial few monolayers, the pseudocubic interlayer is first formed with enhanced c-axis tetragonality $(\mathrm{c} / \mathrm{a}=1.15)$ because of the compressive biaxial lattice strain from the underlying substrate, 
as shown in Fig. 4a. During the growth of the interlayer, partial strain relaxation happens as it is unstable to maintain the same in-plane strain conditions in both $[100]_{\mathrm{p}}$ and $[010]_{\mathrm{p}}$ directions. In this way, two in-plane strain modes exit: a larger compressive strain $\left(\varepsilon_{1}\right)$ is preserved for direction [100] , while a much smaller compressive strain $\left(\varepsilon_{2}\right)$ for direction $[010]_{\mathrm{p}}$ (Fig. 4b). This strain-driven lattice distortion along $[100]_{\mathrm{p}}$ moves up the oxygen ions A between Fe and $\mathrm{Mn}$ and decreases their distance, leading to a smaller distance along $[100]_{\mathrm{p}}\left(d_{a}\right)$ than that along $[010]_{\mathrm{p}}\left(d_{b}\right)$ (Fig. 4c). The c-axis movement of oxygen ions $\mathrm{B}$ along $[010]_{\mathrm{p}}$ becomes much smaller because of the larger strain relaxation in $[100]_{\mathrm{p}}$ direction. The asymmetric movements of A and B oxygen ions form incomplete oxygen octahedra, acting as trapping sites for incoming $\mathrm{Bi}$ adatoms during the film growth. Therefore, at the top of the interlayer, Bi adatoms could be captured on the top center of the pairs of four A ions.

Fig. 4d describes the entire growth process of the SC structure along $[010]_{\mathrm{p}}$ and $[100]_{\mathrm{p}}$ directions based on the atomic models and their corresponding STEM images. In the interlayer region, different growth environments $\left(d_{a}\right.$ and $\left.d_{b}\right)$ along these two directions limit the final positions of $\mathrm{Bi}$ adatoms. In particular, $\mathrm{Bi}$ adatoms tend to stay at the top middle positions of the A ions in direction $[100]_{\mathrm{p}}$, forming a constant one-to-one relationship with $\mathrm{Fe} / \mathrm{Mn}$ ions from the interlayer to the upper SC film. However, in direction $[010]_{\mathrm{p}}$, the narrower spacing of $d_{b}$ attracts extra Fe/Mn ions to stack with original $\mathrm{Bi}$ ions. This unique requirement leads to a $(\mathrm{Fe} / \mathrm{Mn})$ : $\mathrm{Bi}$ lattice matching transition from 1:1 to 4:3, which is accompanied with significant strain relaxation ${ }^{37}$. Once the stable $\mathrm{Bi}_{2} \mathrm{O}_{2}$ layer is formed, the BFMO SC structure is obtained through selfalternative growth of $\mathrm{Bi}_{2} \mathrm{O}_{2}$ layers and $\left[\mathrm{Fe}_{0.5} \mathrm{Mn}_{0.5}\right] \mathrm{O}_{6}$ layers. It should be noticed that a 
small amount of $[010]_{\mathrm{p}}$-oriented SC structures was also observed along the $[001]_{\mathrm{p}}$ zone axis (Supplementary Fig. S4), further confirming that strain relaxations of $\varepsilon_{1}$ and $\varepsilon_{2}$ could both happen on the cubic LAO unit cells and form the resulting incommensurate stacking sequence in different directions.

The above growth model demonstrates the important role of lattice strain and interface reconstruction in triggering the alternating growth of $\mathrm{Bi}_{2} \mathrm{O}_{2}$ layer and $\left[\mathrm{Fe}_{0.5} \mathrm{Mn}_{0.5}\right] \mathrm{O}_{6}$ layer in BFMO SC structure. As the Bi-layered stacking is a relatively stable configuration as seen in many Aurivillius phases, the formation of octahedral plane similar to the $\mathrm{Fe}_{0.5} \mathrm{Mn}_{0.5} \mathrm{O}$ system is critical to obtain new single-phase SC structures. Driven by first-principles calculations, a growth strategy is proposed for exploring new self-assembled SCs based on strain-driven formation of partial $\left[\mathrm{M}_{1-\mathrm{x}} \mathrm{M}_{\mathrm{x}}{ }_{\mathrm{x}}\right] \mathrm{O}_{6}$ octahedral plane (M, M’= Fe, Co, Ni, Mn, Cr).

Based on the above prediction, our first attempt to grow a new SC structure of the Bi-Co-Mn-O (BCMO) system from a single-phase $\mathrm{Bi}_{2} \mathrm{CoMnO}_{6}$ target shows great promise. Fig. 5a shows its high-resolution HAADF STEM image. An interesting layered structure with a self-assembled ultrathin interlayer is observed in BCMO SC films. While sharing the characteristics of using Bi-based unit as the building frame of layered oxide structures, the BCMO SC shows several different features from the BFMO SC film: (i) This interlayer exhibits an enhanced tetragonality $(\mathrm{c} / \mathrm{a}=1.18)$ similar to BFMO SC film (c/a=1.15), but a decreased thickness of $\sim 0.76 \mathrm{~nm}$, as shown in the inset in Fig. 5a; (ii) Beyond the interlayer, an in-plane rotation was identified between the zone axis of the BCMO film and the underlying LAO substrate; (iii) the growth of BCMO SC is based on alternative stacking of one $\left[\mathrm{Co}_{0.5} \mathrm{Mn}_{0.5}\right] \mathrm{O}_{6}$ layer and one $\mathrm{Bi}_{3} \mathrm{O}_{3}$ layer. 
Fig. 5b shows the enlarged STEM image of a tilted BCMO film so that one layer of $\left[\mathrm{Co}_{0.5} \mathrm{Mn}_{0.5}\right] \mathrm{O}_{6}$ is clearly observed with neighboring $\mathrm{Bi}_{3} \mathrm{O}_{3}$ layers. The decreased interlayer thickness, as well as the in-plane rotation of the SC film, indicates a different strain relaxation condition in BCMO film, which possibly results in the different selfalternative stacking mode in forming the BCMO structure. Fig. $5 \mathrm{c}$ shows the corresponding selected area electron diffraction (SAED) pattern of the BCMO film. The c axis lattice parameter is obtained as $\sim 26.1 \AA$, which is about twice of the spacing between the neighboring $\mathrm{Bi}_{3} \mathrm{O}_{3}$ sheets $(\sim 12.9 \AA)$ in STEM and agrees well with the measured value ( 26.2 $\AA$ ) from X-ray diffraction (XRD) result in Fig. 5d.

More interestingly, the first measurements indicate promising room-temperature multiferroic property of the BCMO film as characterized by local ferroelectric/piezoelectric switching measurement and vibrating sample magnetometer test. Under ambient conditions, the BCMO film showed a sharp phase switching regarded as a characteristic of ferroelectric materials with a remnant polarization $\left(P_{r}\right)$ of 0.20 $\mu \mathrm{C} / \mathrm{cm}^{2}$ (Fig. 5e). The smaller $P_{r}$ value compared to the BFMO SC films is possibly related to the different layer stacking of $\mathrm{Bi}_{\mathrm{x}} \mathrm{O}_{\mathrm{x}}$ layers with respect to $\left[\mathrm{Fe}_{0.5} \mathrm{Mn}_{0.5}\right] \mathrm{O}_{6}$ or $\left[\mathrm{Co}_{0.5} \mathrm{Mn}_{0.5}\right] \mathrm{O}_{6}$ octahedra layers, which affects the degree of lattice modulation and spin frustration during the ferroelectric switching ${ }^{44}$. The field-dependent magnetization of the BCMO SC film is shown in Fig. 5f, exhibiting a room temperature saturation magnetization of $\sim 85$ eum $/ \mathrm{cm}^{3}$ comparable to that in BFMO SC structure $(\sim 110$ $\mathrm{emu} / \mathrm{cm}^{3}$ ). The preliminary results, shown in Fig. 5, demonstrate great opportunities in designing Bi-based layered oxide structures with room-temperature multiferroic properties. 
In conclusion, the combined experimental (HAADF and ABF-STEM, EDS and PED) and first-principles calculations reveal the detailed structure and growth mechanism of a new class of self-assembled Bismuth-based two-dimensional (2D) layered oxide structures, later identified with a lattice space group of $\mathrm{P} 2{ }_{1} / \mathrm{m}$ (or simplified Amm2). It suggests the substrate-induced biaxial strain promotes lattice distortion and interface reconstruction in the initial interlayers, and subsequently facilitates the self-assembled layer stacking of the following atomic crystals. The formations of the new class of selfassembled 2D structures confirm the high potential of the strain-driven approach to tailor new multifunctional layered oxide films and provide a significant step towards new materials designs for future high-performance nanoelectronic devices. 


\section{References}

1. Geim, A.K. \& Grigorieva, I.V. Van der Waals heterostructures. Nature 499, 419-425 (2013).

2. Butler, S.Z. et al. Progress, Challenges, and Opportunities in Two-Dimensional Materials Beyond Graphene. ACS Nano 7, 2898-2926 (2013).

3. Novoselov, K.S. et al. Two-dimensional atomic crystals. Proc. Natl. Acad. Sci. 102, 10451-10453 (2005).

4. Yu, P., Chu, Y.-H. \& Ramesh, R. Oxide interfaces: pathways to novel phenomena. Mater. Today 15, 320-327 (2012).

5. Lee, J.H. et al. A strong ferroelectric ferromagnet created by means of spin-lattice coupling. Nature 466, 954-958 (2010).

6. Schlom, D.G. et al. Strain Tuning of Ferroelectric Thin Films. Annu. Rev. Mater. Res. 37, 589-626 (2007).

7. Novoselov, K.S. et al. Electric Field Effect in Atomically Thin Carbon Films. Science 306, 666-669 (2004).

8. Geim, A.K. \& Novoselov, K.S. The rise of graphene. Nat. Mater. 6, 183-191 (2007).

9. Berger, C. et al. Ultrathin Epitaxial Graphite: 2D Electron Gas Properties and a Route toward Graphene-based Nanoelectronics. J. Phys. Chem. B 108, 19912-19916 (2004).

10. Castro Neto, A.H., Guinea, F., Peres, N.M.R., Novoselov, K.S. \& Geim, A.K. The electronic properties of graphene. Rev. Mod. Phys. 81, 109-162 (2009).

11. Castro Neto, A.H. Charge Density Wave, Superconductivity, and Anomalous Metallic Behavior in 2D Transition Metal Dichalcogenides. Phys. Rev. Lett. 86, 4382-4385 (2001).

12. Lu, C.-H., Yang, H.-H., Zhu, C.-L., Chen, X. \& Chen, G.-N. A Graphene Platform for Sensing Biomolecules. Angew. Chem. Int. Ed. 48, 4785-4787 (2009).

13. Sun, Y., Wu, Q. \& Shi, G. Graphene based new energy materials. Energy Environ. Sci. 4, 1113-1132 (2011).

14. Podzorov, V., Gershenson, M.E., Kloc, C., Zeis, R. \& Bucher, E. High-mobility fieldeffect transistors based on transition metal dichalcogenides. Appl. Phys. Lett. 84, 33013303 (2004).

15. Pacilé, D., Meyer, J.C., Girit, Ç.Ö. \& Zettl, A. The two-dimensional phase of boron nitride: Few-atomic-layer sheets and suspended membranes. Appl. Phys. Lett. 92 (2008).

16. Shelimova, L.E. et al. Synthesis and structure of layered compounds in the $\mathrm{PbTe}-\mathrm{Bi}_{2} \mathrm{Te}_{3}$ and $\mathrm{PbTe}-\mathrm{Sb}_{2} \mathrm{Te}_{3}$ systems. Inorg. Mater. 40, 1264-1270 (2004).

17. Coleman, J.N. et al. Two-Dimensional Nanosheets Produced by Liquid Exfoliation of Layered Materials. Science 331, 568-571 (2011).

18. Lee, C.-H. et al. Exploiting dimensionality and defect mitigation to create tunable microwave dielectrics. Nature 502, 532-536 (2013).

19. Takada, K. et al. Superconductivity in two-dimensional $\mathrm{CoO}_{2}$ layers. Nature 422, 53-55 (2003).

20. Yamauchi, H., Sakai, K., Nagai, T., Matsui, Y. \& Karppinen, M. Parent of MisfitLayered Cobalt Oxides: $\left[\mathrm{Sr}_{2} \mathrm{O}_{2}\right]_{4} \mathrm{CoO}_{2}$. Chem. Mater. 18, 155-158 (2005).

21. Li, X. et al. Large-Area Synthesis of High-Quality and Uniform Graphene Films on Copper Foils. Science 324, 1312-1314 (2009).

22. Meyer, J.C. et al. The structure of suspended graphene sheets. Nature 446, 60-63 (2007).

23. Novoselov, K.S. et al. Two-dimensional gas of massless Dirac fermions in graphene. Nature 438, 197-200 (2005).

24. Chen, J.-H., Jang, C., Xiao, S., Ishigami, M. \& Fuhrer, M.S. Intrinsic and extrinsic performance limits of graphene devices on $\mathrm{SiO}_{2}$. Nat. Nanotech. 3, 206-209 (2008). 
25. Zandbergen, H.W., Gronsky, R., Wang, K. \& Thomas, G. Structure of $(\mathrm{CuO})_{2}$ double layers in superconducting $\mathrm{YBa}_{2} \mathrm{Cu}_{3} \mathrm{O}_{7}$. Nature 331, 596-599 (1988).

26. Lake, B. et al. Antiferromagnetic order induced by an applied magnetic field in a hightemperature superconductor. Nature 415, 299-302 (2002).

27. Ramirez, A.P. Colossal magnetoresistance. J. Phys.: Condens. Matter 9, 8171 (1997).

28. Wang, J. et al. Epitaxial $\mathrm{BiFeO}_{3}$ Multiferroic Thin Film Heterostructures. Science 299, 1719-1722 (2003).

29. Chen, A. et al. A New Class of Room-Temperature Multiferroic Thin Films with Bismuth-Based Supercell Structure. Adv. Mater. 25, 1028-1032 (2013).

30. Hwang, H.Y. et al. Emergent phenomena at oxide interfaces. Nat. Mater. 11, 103-113 (2012).

31. Zhang, W. et al. Interfacial coupling in heteroepitaxial vertically aligned nanocomposite thin films: From lateral to vertical control. Curr. Opin. Solid State Mater. Sci. 18, 6-18 (2014).

32. Martin, L.W., Chu, Y.H. \& Ramesh, R. Advances in the growth and characterization of magnetic, ferroelectric, and multiferroic oxide thin films. Mater. Sci. Eng., $R$ 68, 89-133 (2010).

33. Masset, A.C. et al. Misfit-layered cobaltite with an anisotropic giant magnetoresistance: $\mathrm{Ca}_{3} \mathrm{Co}_{4} \mathrm{O}_{9}$. Phys. Rev. B 62, 166-175 (2000).

34. Kendall, K.R., Navas, C., Thomas, J.K. \& zur Loye, H.-C. Recent Developments in Oxide Ion Conductors: Aurivillius Phases. Chem. Mater. 8, 642-649 (1996).

35. Snyder, G.J. \& Toberer, E.S. Complex thermoelectric materials. Nat. Mater. 7, 105-114 (2008).

36. Schaak, R.E. \& Mallouk, T.E. Perovskites by Design: A Toolbox of Solid-State Reactions. Chem. Mater. 14, 1455-1471 (2002).

37. Zhu, Y. et al. Research Updates: Epitaxial strain relaxation and associated interfacial reconstructions: The driving force for creating new structures with integrated functionality. APL Mater. 1 (2013).

38. Okunishi, E. et al. Visualization of Light Elements at Ultrahigh Resolution by STEM Annular Bright Field Microscopy. Microsc. Microanal. 15, 164-165 (2009).

39. Wang, Y., Lv, J., Zhu, L. \& Ma, Y. CALYPSO: A method for crystal structure prediction. Comput. Phys. Commun. 183, 2063-2070 (2012).

40. Lu, P., Zhou, L., Kramer, M.J. \& Smith, D.J. Atomic-scale Chemical Imaging and Quantification of Metallic Alloy Structures by Energy-Dispersive X-ray Spectroscopy. Sci. Rep. 4 (2014).

41. Vincent, R. \& Midgley, P.A. Double conical beam-rocking system for measurement of integrated electron diffraction intensities. Ultramicroscopy 53, 271-282 (1994).

42. Boullay, P. et al. Structure determination of a brownmillerite $\mathrm{Ca}_{2} \mathrm{Co}_{2} \mathrm{O}_{5}$ thin film by precession electron diffraction. Phys. Rev. B 79, 184108 (2009).

43. Palatinus, L. \& Chapuis, G. SUPERFLIP - a computer program for the solution of crystal structures by charge flipping in arbitrary dimensions. J. Appl. Crystallogr. 40, 786-790 (2007).

44. Kimura, T. et al. Magnetic control of ferroelectric polarization. Nature 426, 55-58 (2003). 


\section{Acknowledgements}

This work was supported by the U.S. National Science Foundation (Ceramic Program, NSF-1007969 and 1401266 (Film growth) and NSF-0846504 (High resolution STEM analysis). M. L. and Z. X. acknowledge the support partially from AFOSR MURI (FA9550-12-1-0037). The work at Brookhaven National Laboratory was supported by the US Department of Energy, Office of Basic Energy Science, Division of Materials Science and Engineering, under Contracts no. DE-AC02-98CH10886. )). A portion of the electron microscopy experiments were performed at National Center for Electron Microscopy (NCEM), which is supported by the Office of Science, Office of Basic Energy Sciences of the US Department of Energy under Contract No. DE-AC0205CH11231. W.Z. is grateful to Drs. Peter Ercius, Jim Ciston and Chengyu Song for additional help and fruitful discussions at NCEM.

\section{Author contributions}

H.W. and W.Z. conceived the project and designed the experiment. W.Z. and L. L. grew the BCMO film and conducted the XRD, ferroelectric and magnetic measurements. M.L. and Z.X. conducted the crystal structure analysis by first-principles calculations. W.Z., A.C., Y.Z., H.Z. and J.N. performed the STEM HAADF measurement and image analysis. L.W. and Y.M. performed the STEM ABF measurement. P.L. investigated the crystal structure with the EDS mapping technique. P.B. performed the PED experiment and analysis. W.Z. drafted the manuscript together with A.C. and M.L.; H.W., Q.J., J.M. and X.Z. revised the manuscript. All authors discussed the results and commented on the manuscript. 


\section{Additional information}

Supplementary information included.

\section{Competing financial interests}

The authors declare no competing financial interests. 


\section{Figure Legends}

Figure 1 | Microstructure and oxygen positions of BFMO SC films. (a) STEM HAADF image and (b) STEM ABF image of BFMO SC along the [100] $]_{\mathrm{p}}$ zone axes. (c) Enlarged images of the marked rectangular area in (b). The corresponding intensity profiles identify the positions of stoichiometric oxygen ions (filled circles) and oxygen vacancies (voided circles). Stars represent the cation columns. (e,f) High-resolution STEM ABF images of the SC structure along the $[100]_{p}$ and $[010]_{p}$ zone axes. (d,g) The atomic modeling of BFMO SC structure along the $[100]_{p}$ and $[010]_{p}$ zones axes. (h) BFMO SC crystal structure used in first-principles calculations.

Figure 2 | Atomic EDS mapping of BFMO SC films. (a,f) High-resolution STEM image along the $[100]_{\mathrm{p}}$ zone axis. (b-d) and (g-i) EDS mapping of $\mathrm{Bi}(\mathrm{L}+\mathrm{M}), \mathrm{Mn}\left(\mathrm{K}_{\alpha}\right)$, $\mathrm{Fe}\left(\mathrm{K}_{\alpha}\right)$ along the $[100]_{\mathrm{p}}$ and $[010]_{\mathrm{p}}$ zone axis, respectively. (e,j) Composite color mapping of $\mathrm{Bi}$ (red), $\mathrm{Mn}$ (green) and Fe (blue), respectively. The $\mathrm{Bi}_{2} \mathrm{O}_{2}$ layer is marked out with a triangle, and the $\left[\mathrm{Fe}_{0.5} \mathrm{Mn}_{0.5}\right] \mathrm{O}_{6}$ octahedra layer is represented with a rhombus.

Figure 3 | Crystal structure of BFMO SC films. (a) 0kl and (b) $1 \mathrm{kl}$ reciprocal space sections reconstructed from the PED experiment. The grids are a guide to the eyes, where the reflections coming from the substrate are encircled in red. (c) Different primitive cells and (d) lattice parameters determined by first-principles calculations (Monoclinic) and PED experiments (Orthorhombic). The values in the parentheses represent their correspond ones in the orthorhombic system.

Figure 4 | Strain-driven growth of the SC structure. (a) Schematic model of the interlayer showing enhanced c-axis tetragonality due to the biaxial strain from the substrate. (b) Partial strain relaxation in $[010]_{p}$ and $[100]_{p}$ directions resulting in different movements of A and B oxygen ions. (c) Strain-induced formation of distorted oxygen octahedra as a growth plane for the SC phase. The asymmetric movements of A and B atoms form incomplete oxygen octahedra, acting as trapping sites for incoming $\mathrm{Bi}$ adatoms to grow $\mathrm{Bi}_{2} \mathrm{O}_{2}$ layers. (d) Atomic modeling and corresponding STEM images along the $[010]_{\mathrm{p}}$ and $[100]_{\mathrm{p}}$ direction illustrating the SC growth from the interlayer formation to the alternative layered stacking. 
Figure 5 | Multiferroic BCMO SC films predicted by first-principles calculations. (a) STEM HAADF image of BCMO films on the LAO (001) substrate. Inset shows the enlarged view of the interlayer structure with a thickness of $\sim 7.6 \AA$. (b) Higher magnification STEM HAADF image of the BCMO SC structure showing the alternative stacking of one $[\mathrm{Co} / \mathrm{Mn}] \mathrm{O}_{6}$ layer and one $\mathrm{Bi}_{3} \mathrm{O}_{3}$ layer. (c) Corresponding SAED pattern and (d) XRD pattern of the BCMO structure on LAO (001) substrates. (e) Local piezoelectric phase hysteresis loop of a $400 \mathrm{~nm}$ thick BCMO film. Inset is the ferroelectric polarization hysteresis loop measured at $300 \mathrm{~K}$ and $1 \mathrm{kHz}$. (f) Out-of-plane and in-plane magnetization hysteresis loop of the BCMO film measured at $300 \mathrm{~K}$. 


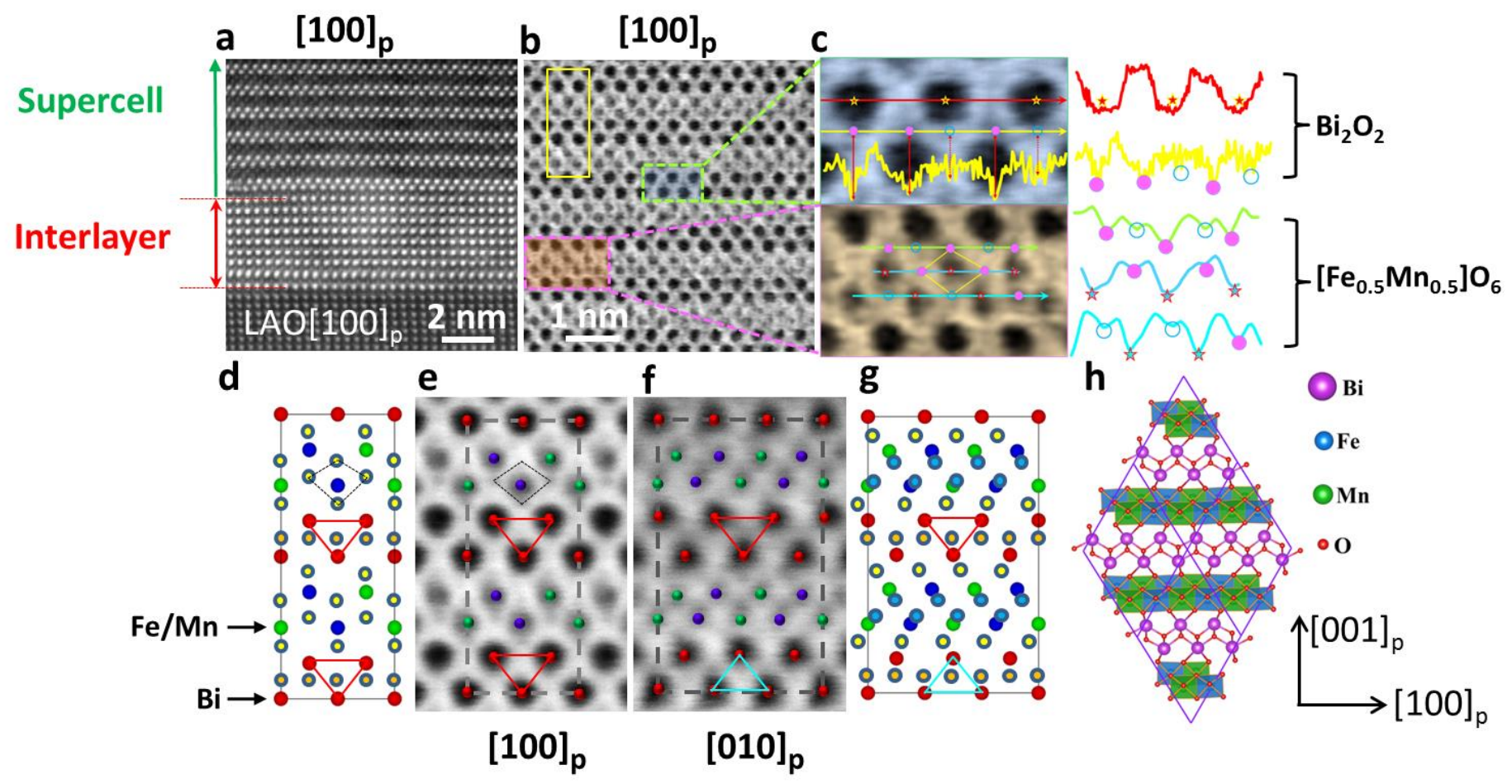




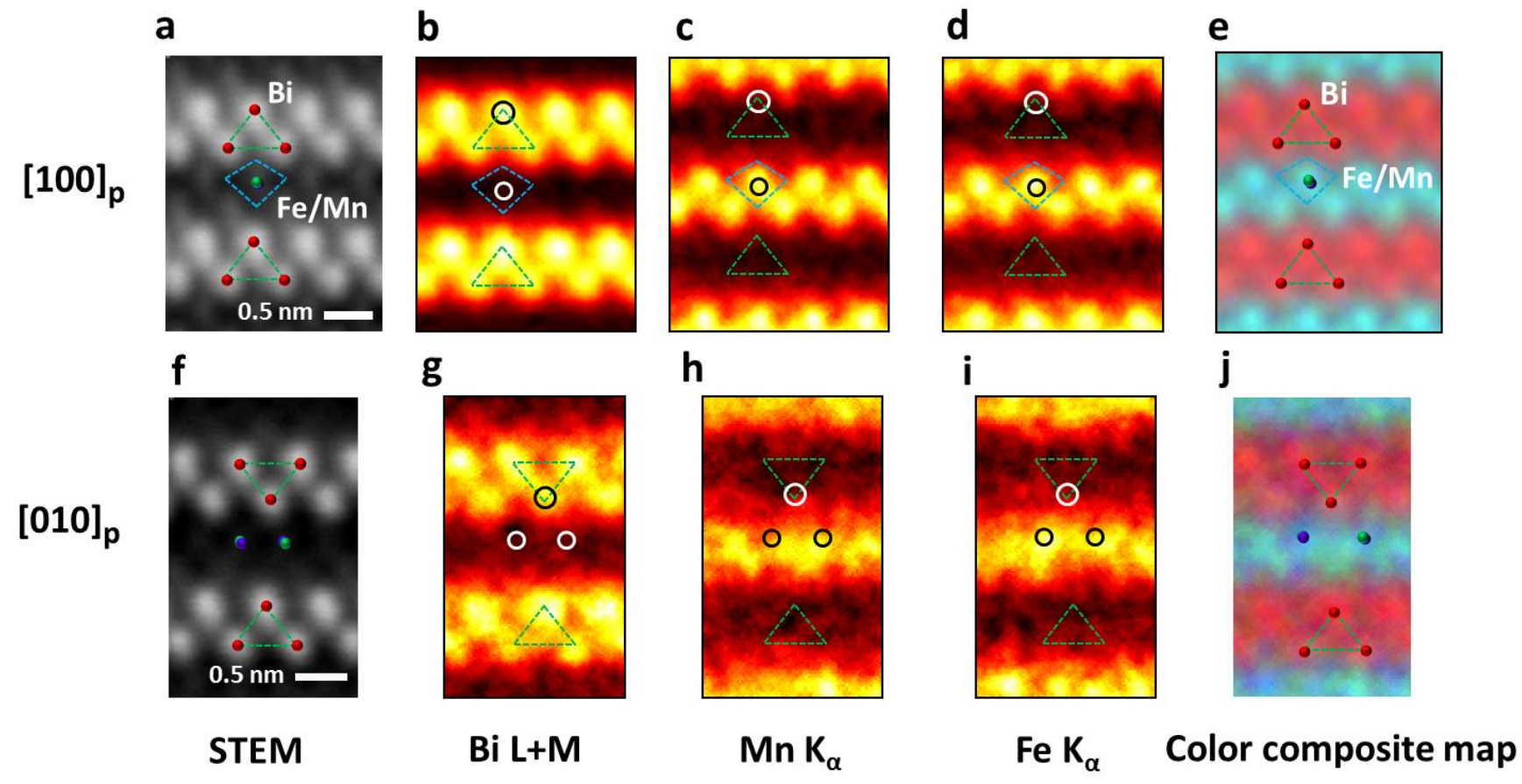



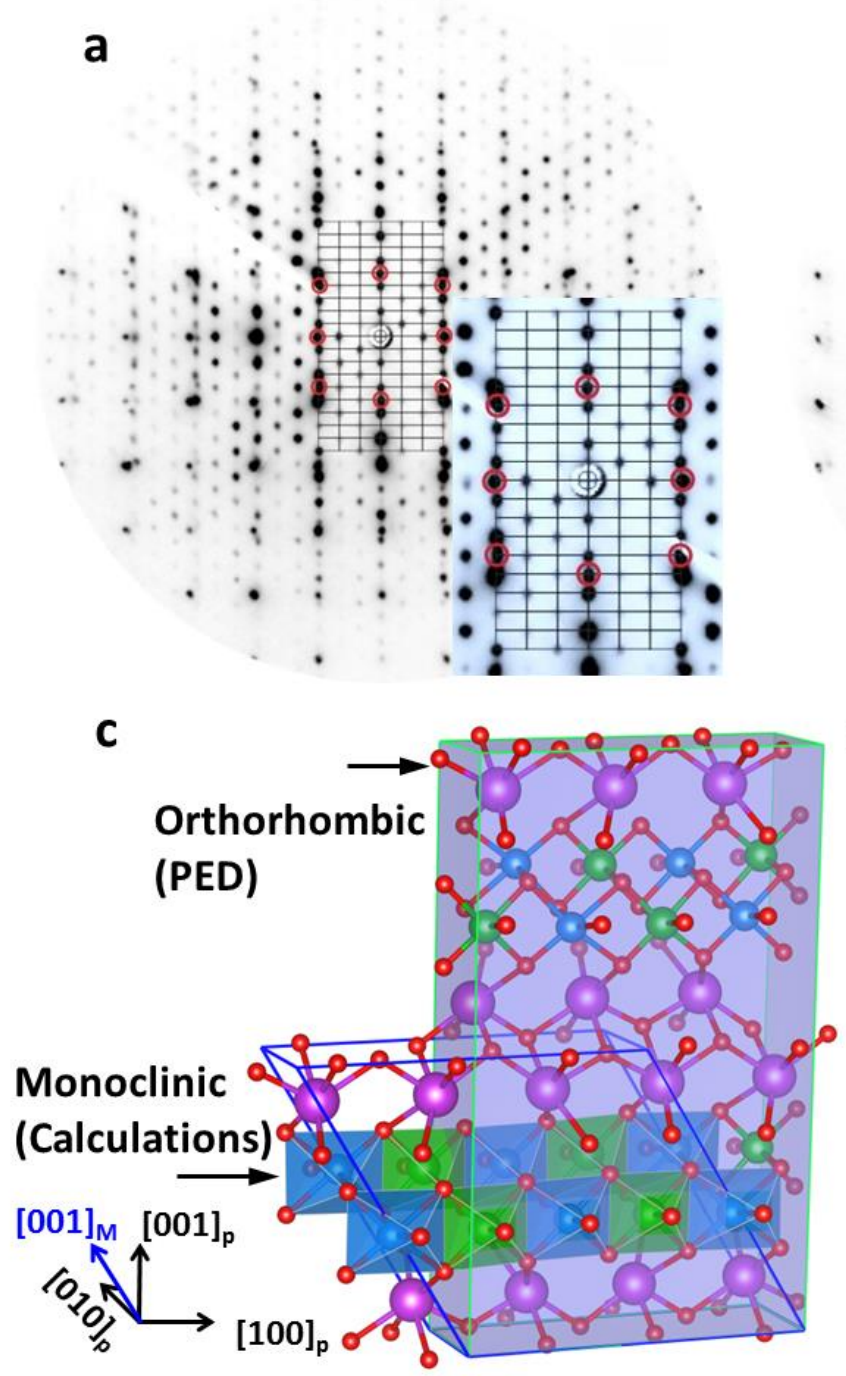

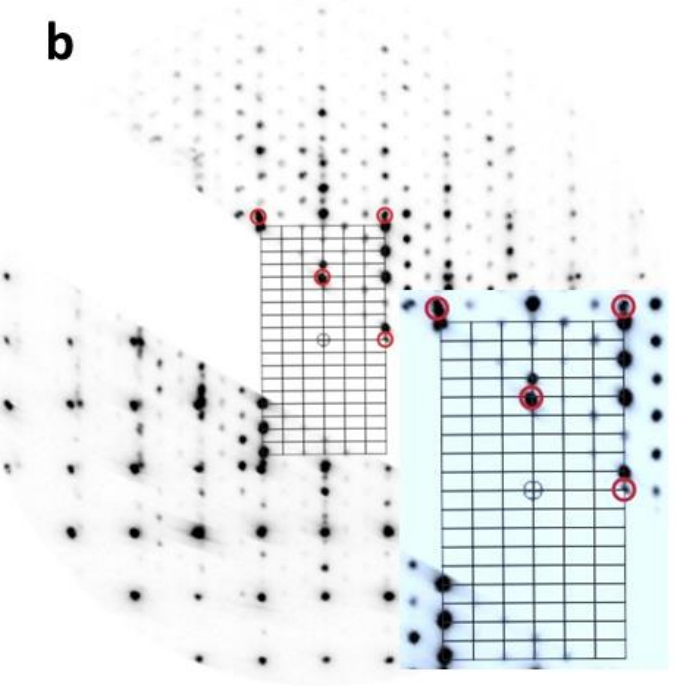

d

\begin{tabular}{|c|c|c|}
\hline & Calculations & PED \\
\hline Space group & $\begin{array}{l}\text { Monoclinic } \\
\mathrm{P2}_{1} / \mathrm{m} \text { (No. 11) }\end{array}$ & $\begin{array}{l}\text { Orthorhombic } \\
\text { Amm2 (No. 38) }\end{array}$ \\
\hline $\mathbf{a}(\AA ̊)$ & 11.8790 & 11.78 \\
\hline b $(\AA ̊)$ & 3.9825 & 3.96 \\
\hline$c\left(c^{\prime}\right)(\AA ̊)$ & $11.4142(19.67)$ & (19.37) \\
\hline$\alpha\left({ }^{\circ}\right)$ & 90 & 90 \\
\hline$\beta\left(\beta^{\prime}\right)\left({ }^{\circ}\right)$ & $120.53(89.18)$ & (90) \\
\hline$\gamma\left({ }^{\circ}\right)$ & 90 & 90 \\
\hline$V\left(V^{\prime}\right)\left(\AA^{3}\right)$ & $465.12(930.24)$ & (903.59) \\
\hline
\end{tabular}



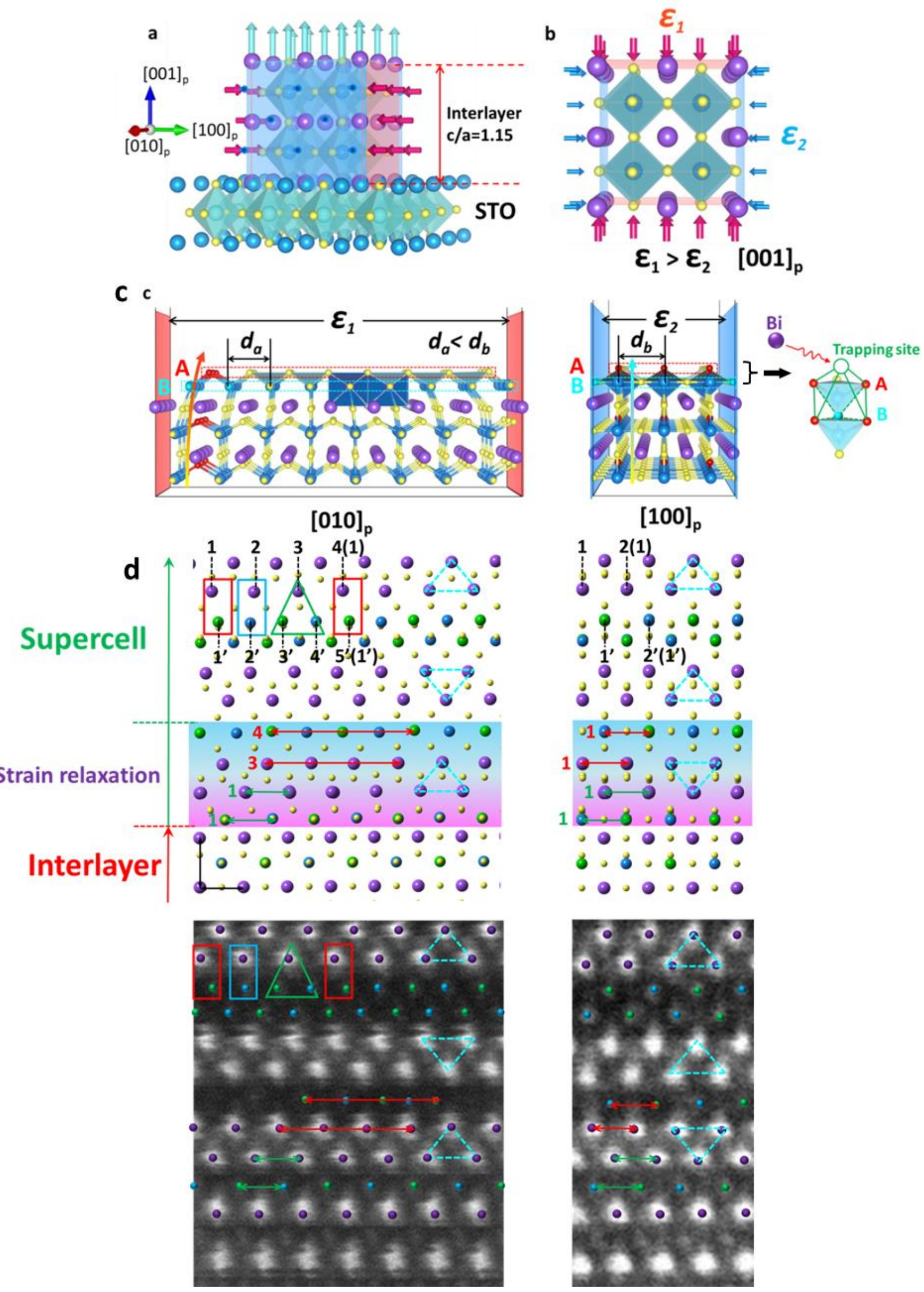

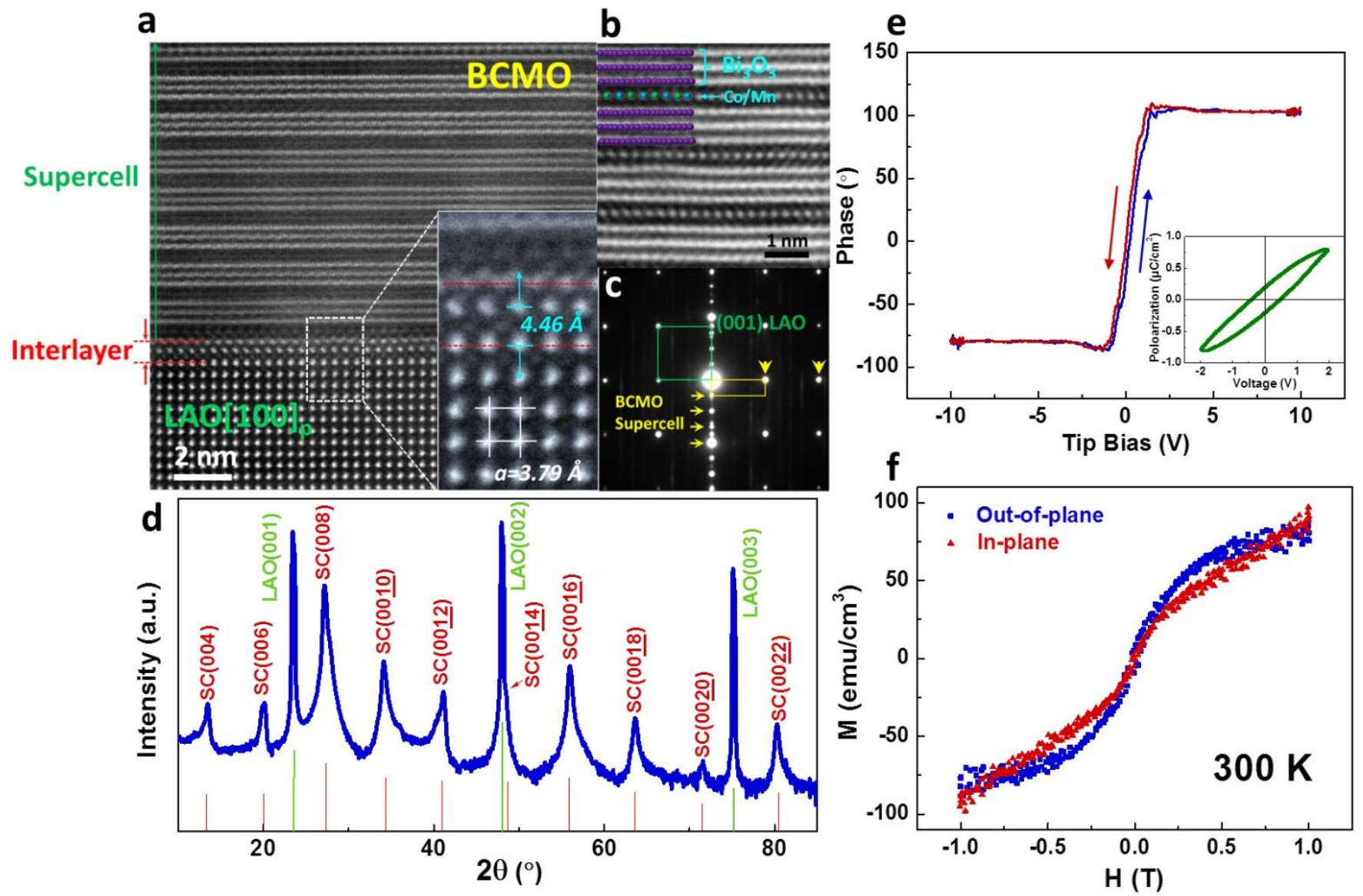


\section{SUPPLEMENTARY INFORMATION}

\section{Two-Dimensional Layered Oxide Structures Tailored by Self- Assembled Layer Stacking via Interfacial Strain}

\footnotetext{
Wenrui Zhang ${ }^{1 \dagger}$, Aiping Chen ${ }^{2 \dagger}$, Mingtao $\mathrm{Li}^{3,4 \dagger}{ }^{3}$, Leigang $\mathrm{Li}^{1}$, Yuanyuan Zhu ${ }^{1}$, Zhenhai Xia ${ }^{3}$, Ping Lu ${ }^{5}$, Philippe Boullay ${ }^{6}$, Lijun Wu ${ }^{7}$, Yimei Zhu ${ }^{7}$, Judith L. MacManus-Driscoll ${ }^{8}$, Quanxi, Jia ${ }^{9}$, Honghui Zhou $^{10}$, Jagdish Narayan ${ }^{10}$, Xinghang Zhang ${ }^{1,11}$, Haiyan Wang ${ }^{1,2 \star}$

${ }^{1}$ Department of Materials Science and Engineering, Texas A and M University, 3128 TAMU, College Station, Texas 77843, USA,

${ }^{2}$ Department of Electrical and Computer Engineering, Texas A and M University, 3128 TAMU, College Station, Texas 77843, USA,

${ }^{3}$ Department of Materials Science and Engineering, and Department of Chemistry, University of North Texas, Denton, Texas 76203, USA,

${ }^{4}$ International Research Center for Renewable Energy, State key Laboratory of Multiphase Flow in Power Engineering, Xi'an Jiaotong University, Xi'an, Shaanxi 710049, P. R. China,

${ }^{5}$ Sandia National Laboratories, Albuquerque, New Mexico 87185, USA

${ }^{6}$ CRISMAT, CNRS UMR 6508, ENSICAEN, 6 Boulevard Maréchal Juin, F-14050 Caen Cedex 4, France,

${ }^{7}$ Condensed Matter Physics \& Materials Science Department, Brookhaven National Laboratory, Upton, New York 11973, USA,

${ }^{8}$ Department of Materials Science, University of Cambridge, Cambridge, CB2 3QZ, UK,

${ }^{9}$ Center for Integrated Nanotechnologies, MS K771, Los Alamos National Laboratory, Los Alamos, New Mexico 87545, USA,

${ }^{10}$ Department of Materials Science and Engineering, NSF Center for Advanced Materials and Smart Structures, North Carolina State University, Raleigh, NC 27695, USA

${ }^{11}$ Department of Mechanical Engineering, Texas A and M University, 3128 TAMU, College Station, Texas 77843, USA.
}

*E-mail: wangh@ece.tamu.edu

${ }^{\dagger}$ These authors contributed equally to this work 


\section{Section I}

\section{Methods}

\section{Sample Preparation}

Epitaxial BFMO and BCMO SC films were deposited on $\mathrm{LaAlO}_{3}$ (001) singlecrystal substrates by pulsed laser deposition (PLD, Lambda Physik, KrF, $\lambda=248 \mathrm{~nm}$ ) from single phase $\mathrm{Bi}_{2} \mathrm{FeMnO}_{6}$ and $\mathrm{Bi}_{2} \mathrm{CoMnO}_{6}$ targets, respectively. The laser fluence was $3 \mathrm{~J} \mathrm{~cm}^{-2}$. The substrate temperature ranged from $650 \sim 700{ }^{\circ} \mathrm{C}$ and a dynamic oxygen pressure of 50 200 mTorr was maintained during depositions. After deposition, the films were in-situ annealed at $400{ }^{\circ} \mathrm{C}$ in 500 Torr of oxygen for 1 hour and then cooled down to $20^{\circ} \mathrm{C}$ at a cooling rate of $5{ }^{\circ} \mathrm{C} / \mathrm{min}$.

\section{XRD, STEM HAADF and ABF Imaging}

The microstructures of as-prepared films were characterized by high-resolution X-ray diffraction (XRD, PANalytical Empyrean). The Z-constrast scanning transmission electron microscopy (STEM) images in high angle annular dark-field (HAADF) mode were obtained by using a VG HB603U STEM equipped with Nion aberration corrector operating at $300 \mathrm{kV}$. The STEM annular bright field (ABF) imaging was conducted in the following conditions: convergent angle $=21.2 \mathrm{mrad}$, collection angle $=8.25-16.8 \mathrm{mrad}$, Cs $=900 \mathrm{~nm}$, focus $=1 \mathrm{~nm}$.

\section{EDS Mapping}

For atomic-scale energy-dispersive X-ray spectroscopy (EDS) chemical mapping, a FEI Titan G2 80-200 STEM with a Cs probe corrector and ChemiSTEM technology (X-FEG and SuperX EDS with four windowless silicon drift detectors) operated at 200 
$\mathrm{kV}$ was used. EDS spectral images were acquired as a series of frames, where the same region was scanned multiple times. Frames were spatially drift-corrected to build up spectral image data using a reference HAADF image. The instantaneous dwell time on each pixel was $100 \mathrm{msec}$, and a typical frame size was 256x256 pixels. Spectral image collection typically took about 1800 sec, yielding a total per-pixel dwell time of about 25 msec. Elemental maps were extracted from spectral image with selected EDS energy widows for each element. To improve the signal-to-noise $(\mathrm{S} / \mathrm{N})$ ratio, a smaller EDS region within the EDS map extracted from a single spectral imaging collection was selected, and an averaged map for this region was obtained by averaging several EDS maps related to each other by the lattice vector translations in the image plane.

\section{PED Tomography}

Precession electron diffraction (PED) tomography data were collected with a precession angle of 1.2 degree on a TEM JEOL2010 (200 kV) equipped with an uppermounted Gatan Orius CCD camera. The PED data collection is performed using the tomography approach proposed by Kolb et al ${ }^{1}$. The precession electron diffraction tomography (PEDT) data were processed using the programs PETS ${ }^{2}$ and Jana2006 ${ }^{3}$ following a procedure similar to the one described in a previous report ${ }^{4}$. The output of the charge flipping procedure is a scattering density map (electrostatic potential in the current case) that can be interpreted in terms of atomic positions. The obtained structural model was refined against the PED data and completed using Fourier difference maps leading to the structure in Table S2. The PED data are biased by the dynamical diffraction effects still present even using the precession method and leading 
to high values of reliability factors (Robs around 30\%). To maintain reasonable geometry of the $[\mathrm{Mn} / \mathrm{Fe}] \mathrm{O}_{6}$ octahedra, soft restraints on atomic distances were imposed.

\section{First-principles Calculations}

In order to determine the position of the oxygen atoms, an unbiased search of the global minimum (GM) structures of $\mathrm{Bi}_{6} \mathrm{Fe}_{4} \mathrm{Mn}_{4} \mathrm{O}_{10+} \delta$ was carried out from firstprinciples calculations employing Crystal structure AnaLYsis by Particle Swarm Optimization (CALYPSO) code ${ }^{5}$ with cation constraints. The underlying ab initio local structural relaxations and total energy calculations were performed in the framework of density functional theory within the generalized gradient approximation (GGA) with the Perdew-Burke-Enzerhof parameterization (PBE) ${ }^{6}$ and projector-augmented wave (PAW) method $^{7,8}$, as implemented in the VASP code ${ }^{9,10}$. For the obtained low energy structures, further DFT calculations were performed with the Nuclei-electron interactions described by PAW, the electronic exchange and correlation effects described by PBE. The Bi$5 d^{10} 6 s^{2} 6 p^{3}$, Fe- $3 d^{6} 4 s^{2}, M n-2 p^{6} 3 d^{5} 4 s^{2}, O-2 s^{2} 2 p^{4}$ were treated as valence electrons. In order to calculate the oxygen vacancies position, a $1 \times 2 \times 1$ supercell was employed thought out all the calculations except marked explicitly. The bulk lattice parameters of $\mathrm{Bi}_{3} \mathrm{Fe}_{2} \mathrm{Mn}_{2} \mathrm{O}_{10-\delta}$ were relaxed and optimized by using $640 \mathrm{eV}$ as the cutoff energy for basis function and $3 \times 4 \times 3$ Monkhorst-Pack-type kpoint sampling centered at the gamma ( $\Gamma)$ point, which were benchmarked to be sufficient to reach the convergence for the calculations. The Fermi level was slightly broadened using a Fermi-Dirac smearing of $100 \mathrm{meV}$. All calculations were spin polarized with a force convergence criterion of $2 \times 10^{-2} \mathrm{eV} / \AA$. In order to consider the on-site Coulomb interaction of the localized $d$ 
electrons of $\mathrm{Fe}$ and $\mathrm{Mn}, \mathrm{PBE}+\mathrm{U}$ calculations were also performed using the Hubbard model with effective interaction parameter $\mathrm{U}_{\text {eff }}$ 4.6/4.0 eV for Fe/Mn.

\section{Electrical and Magnetic Property Measurement}

The magnetic property of as-prepared BCMO films were investigated by the vibrating sample magnetometer (VSM) mode in a Quantum Design Physical Properties Measurement System (PPMS 6000). The out-of-plane magnetizations were measured by applying a magnetic field perpendicular to the film plane, and the in-plane magnetizations were tested by applying the field parallel to the film plane. The substrate signals have been subtracted for all measured samples. In order to characterize the ferroelectric/piezoelectric properties of BCMO films, a thin $\mathrm{CeO}_{2} / \mathrm{SrRuO}_{3}$ buffer was first in-situ deposited on LAO (001) substrate. Gold contact with the diameter of $350 \mu \mathrm{m}$ was then deposited on top of BCMO films by magnetron sputtering to fabricate a thin film capacitor of $\mathrm{Au}(100 \mathrm{~nm}) / \mathrm{BFCO}(400 \mathrm{~nm}) / \mathrm{CeO}_{2}(15 \mathrm{~nm}) / \mathrm{SrRuO}_{3}(15 \mathrm{~nm}) / \mathrm{LAO}(001)$. The as-prepared capacitor was then tested with piezoresponse force microscopy measurements (Veeco Dimension NanoMan) using a Pt/Ir-coated tip and ferroelectric switching experiments (TF Analyzer 1000). 


\section{Section II}

Structural information of primitive cells determined by first-principles calculations

Table S1 Structure information of $\mathrm{Bi}_{3} \mathrm{Fe}_{2} \mathrm{Mn}_{2} \mathrm{O}_{11}$ from PBE4.6/4.0 (Space group P2 $1 / \mathrm{m}$ (No. 11), $a=11.8790 \AA, b=3.9825 \AA, c=11.4142 \AA, \alpha=\gamma=90^{\circ}, \beta=120.53^{\circ}$ )

\begin{tabular}{|c|c|c|c|}
\hline Atom & $\mathbf{x}$ & $\mathbf{y}$ & $\mathbf{z}$ \\
\hline $\operatorname{Bi1}(2 e)$ & 0.0212 & 0.25 & 0.8723 \\
\hline $\operatorname{Bi} 2(2 e)$ & 0.3563 & 0.25 & 0.8669 \\
\hline $\operatorname{Bi} 3(2 e)$ & 0.6840 & 0.25 & 0.8748 \\
\hline Fe(Mn)1(2e) & 0.0094 & 0.25 & 0.4012 \\
\hline $\mathrm{Fe}(\mathrm{Mn}) 2(2 e)$ & 0.2680 & 0.25 & 0.4046 \\
\hline $\mathrm{Fe}(\mathrm{Mn}) 3(2 e)$ & 0.5203 & 0.25 & 0.4048 \\
\hline $\mathrm{Fe}(\mathrm{Mn}) 4(2 e)$ & 0.7708 & 0.25 & 0.4163 \\
\hline $01(2 e)$ & 0.0786 & 0.25 & 0.2724 \\
\hline O2(2e) & 0.2147 & 0.25 & 0.5460 \\
\hline O3(2e) & 0.2510 & 0.25 & 0.9906 \\
\hline 04(2e) & 0.3296 & 0.25 & 0.2755 \\
\hline O5(2e) & 0.4551 & 0.25 & 0.5460 \\
\hline $06(2 e)$ & 0.5814 & 0.25 & 0.0031 \\
\hline O7(2e) & 0.5875 & 0.25 & 0.2811 \\
\hline $08(2 e)$ & 0.7212 & 0.25 & 0.5601 \\
\hline O9(2e) & 0.8229 & 0.25 & 0.2802 \\
\hline $010(2 e)$ & 0.9040 & 0.25 & 0.9780 \\
\hline
\end{tabular}




\begin{tabular}{llll}
\hline O11(2e) & 0.9609 & 0.25 & 0.5545 \\
\hline
\end{tabular}



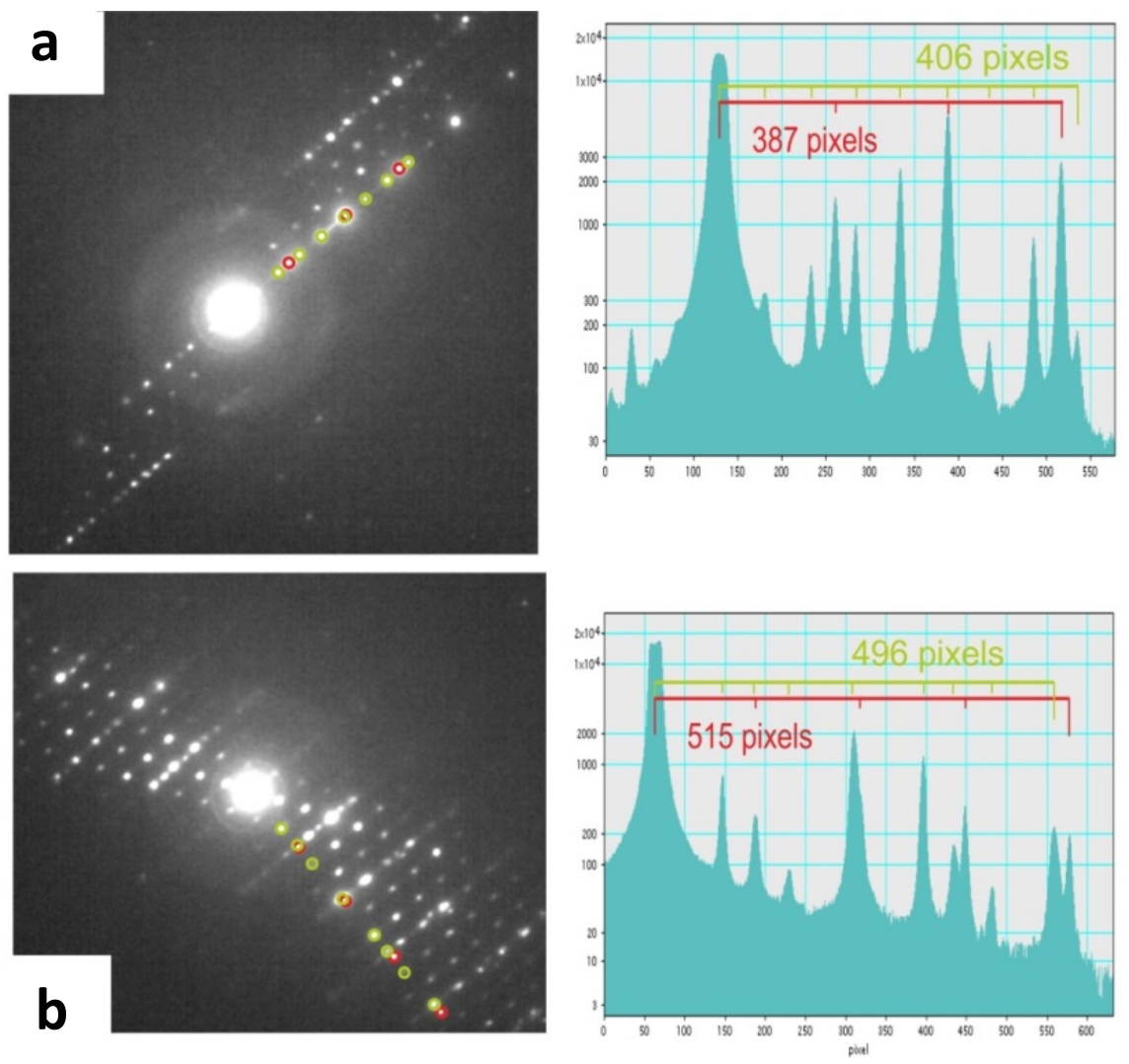

Figure S1 (a) and (b) part of two patterns taken during the tomography experiment. They both indicate that there is a lattice mismatch between the BFMO SC structure (in green) and the LAO substrate (in red). 
a

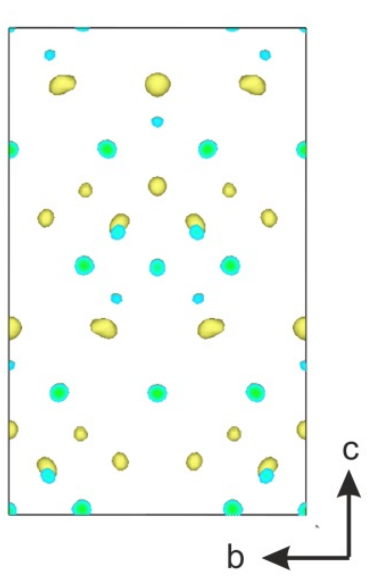

d

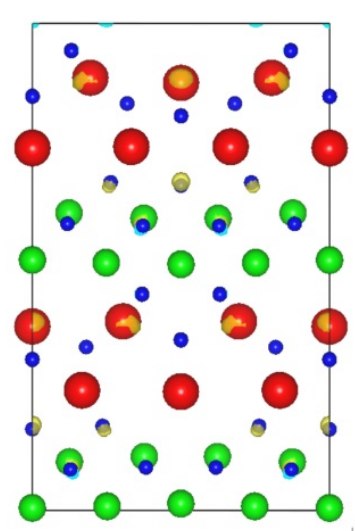

b

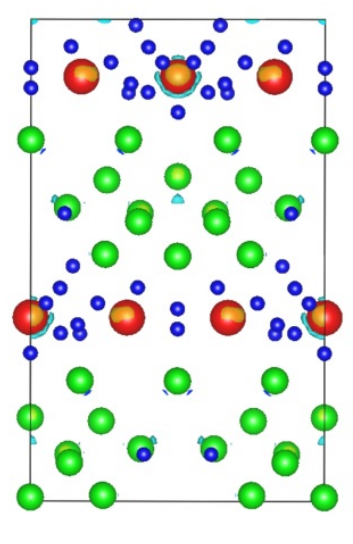

e

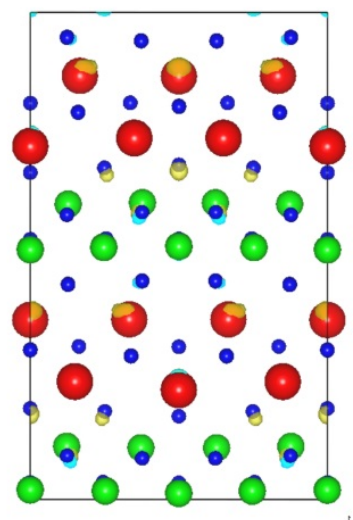

C

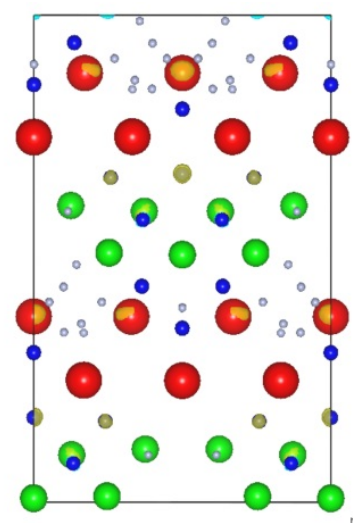

f

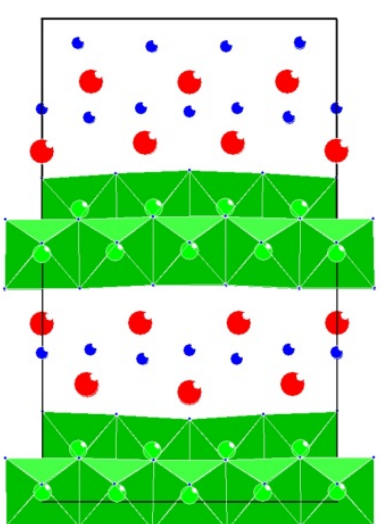

Figure S2 (a) $[010]_{\mathrm{p}}$ projection of the electron density map generated by the Superflip as an output of the charge-flipping structure solution procedure. (b) Raw solution obtained from Superflip superimposed to the electron density map. $\mathrm{Bi}, \mathrm{Mn} / \mathrm{Fe}$ and $\mathrm{O}$ atoms are represented in red, green and blue, respectively. (c) Interpretation of the raw solution (grey positions are considered as ghosts). (d) Atomic positions after refinement and use of Fourier difference calculation. (e) and (f) Proposed refined model after adding some oxygen atoms and distance restraints. 


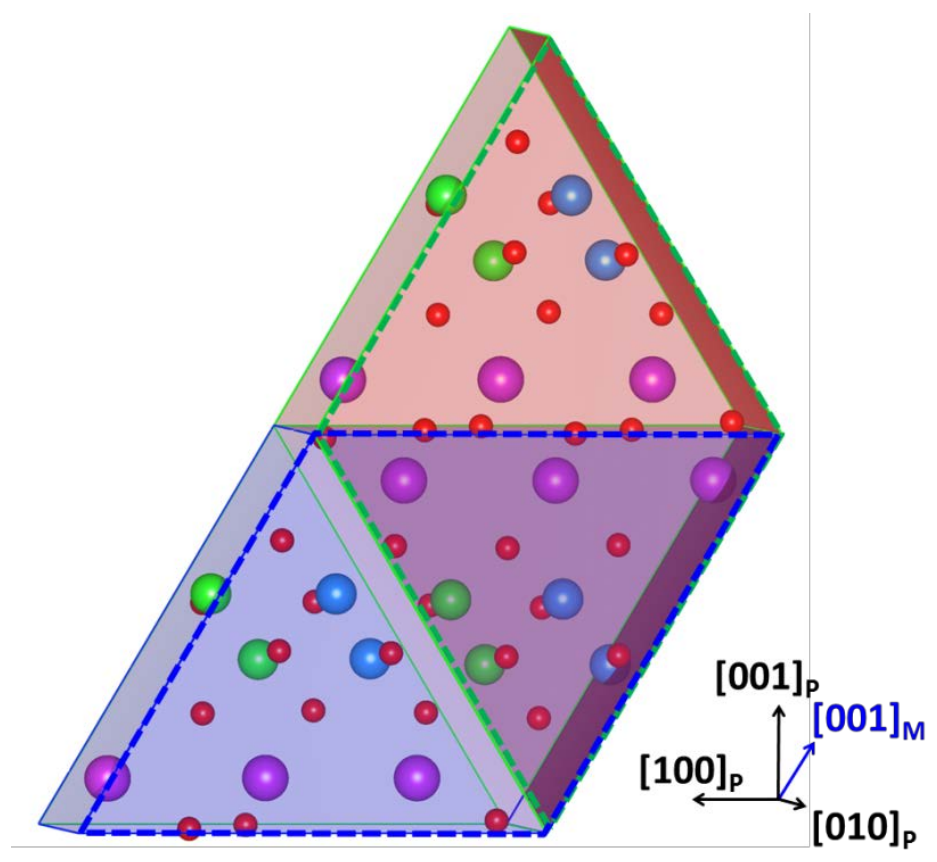

Figure S3 Crystal structure models selected for oxygen lattice site calculation (in green) and for layered stacking explanation (in blue). It is clear that these two models represent the same structure information of the entire BFMO supercell. 
Table S2 Structure information of BFMO SC from PED data

(Space group Amm2 (No. 38), a=11.78 $\AA$, $b=3.96 \AA, c=19.37 \AA, \alpha=\beta=\gamma=90^{\circ}$ )

\begin{tabular}{|c|c|c|c|c|}
\hline Atom & $x$ & $y$ & $\mathbf{Z}$ & Uiso $\left(\AA^{2}\right)$ \\
\hline Bi1 & 0.5 & 0 & $0.3686(5)$ & $0.0183(9)$ \\
\hline Bi2 & 0.5 & $0.3333(5)$ & $0.3697(5)$ & $0.0183(9)$ \\
\hline Bi3 & 0 & $0.1501(5)$ & $0.2421(5)$ & $0.0183(9)$ \\
\hline Bi4 & 0 & 0.5 & $0.2251(5)$ & $0.0183(9)$ \\
\hline$(\mathrm{Mn}, \mathrm{Fe}) 1$ & 0.5 & $0.1227(10)$ & $0.1092(6)$ & $0.0146(12)$ \\
\hline$(\mathrm{Mn}, \mathrm{Fe}) 2$ & 0 & $0.2471(10)$ & $0.5178(6)$ & $0.0146(12)$ \\
\hline$(\mathbf{M n}, \mathbf{F e}) 3$ & 0.5 & $0.3727(10)$ & $0.1062(6)$ & $0.0146(12)$ \\
\hline (Mn,Fe)4 & 0 & 0.5 & $0.5201(8)$ & $0.0146(12)$ \\
\hline$(\mathrm{Mn}, \mathrm{Fe}) 5$ & 0 & 0 & $0.5129(8)$ & $0.0146(12)$ \\
\hline 01 & 0.5 & 0 & $0.1871(11)$ & $0.032(3)$ \\
\hline $\mathbf{O} 2$ & 0 & $0.3756(16)$ & $0.5905(10)$ & $0.032(3)$ \\
\hline O3 & 0.5 & $0.2507(17)$ & $0.1810(9)$ & $0.032(3)$ \\
\hline 04 & 0 & 0 & $0.3074(14)$ & $0.032(3)$ \\
\hline O5 & 0 & $0.3763(11)$ & $0.4487(10)$ & $0.032(3)$ \\
\hline O7 & 0 & $-0.124(2)$ & $0.5849(13)$ & $0.032(3)$ \\
\hline O6 & 0 & $0.3389(19)$ & $0.2948(12)$ & $0.032(3)$ \\
\hline 08 & 0.5 & $0.1637(13)$ & $0.3140(10)$ & $0.032(3)$ \\
\hline O9 & 0.5 & 0.5 & $0.3140(18)$ & $0.032(3)$ \\
\hline 010 & 0.5 & 0.5 & $0.1717(13)$ & $0.032(3)$ \\
\hline
\end{tabular}




\begin{tabular}{ccccc}
\hline $\mathbf{0 1 1}$ & 0.5 & 0 & $0.5341(12)$ & $0.032(3)$ \\
$\mathbf{0 1 2}$ & 0.5 & $0.2549(16)$ & $0.5352(10)$ & $0.032(3)$ \\
$\mathbf{0 1 3}$ & 0.5 & 0.5 & $0.5345(13)$ & $0.032(3)$ \\
$\mathbf{0 1 4}$ & 0 & $-0.1270(14)$ & $0.4410(9)$ & $0.032(3)$ \\
\hline
\end{tabular}

PED results of the refinement of BFMO SC from PED data consider the space group Amm2 with the cell parameters a=3.96 $\AA, b=11.78 \AA$ and $c=19.37 \AA$. Isotropic thermal displacement parameters (Uiso) are refined to a same value for $\mathrm{Bi},(\mathrm{Mn}, \mathrm{Fe})$ and $\mathrm{O}$ atoms respectively. The number of reflections used in the refinement with $\mathrm{I}>3 \sigma(\mathrm{I})$ is 1688 averaged from 4038 reflections. The reliability factors Robs and wRobs are, respectively, $30.8 \%$ and $36.7 \%$. 


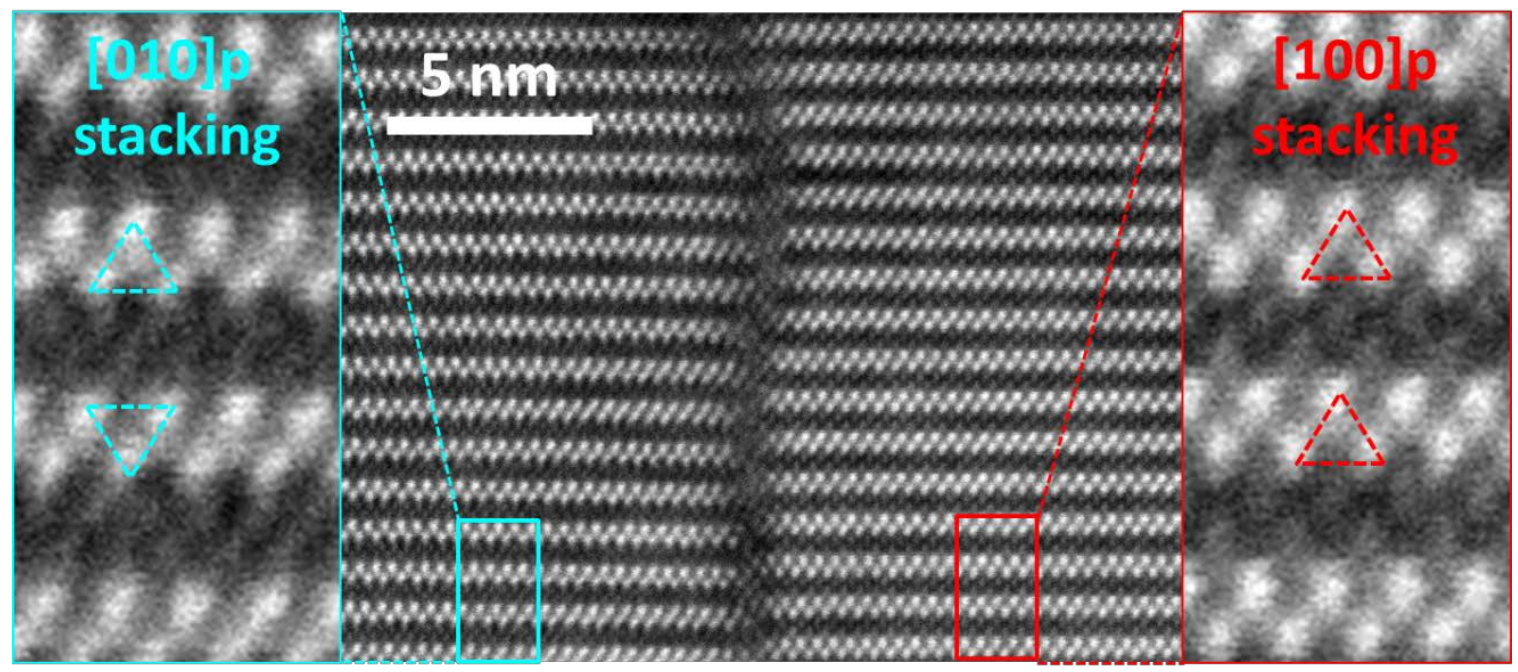

\section{[100]p zone axis}

Figure S4 HAADF STEM image of a grain boundary region showing both $[100]_{\mathrm{p}}$ and $[010]_{\mathrm{p}}$ stacking sequence along the [100]p zone axis. The presence of these two stacking sequences along the LAO[001] direction indicates the strain relaxation of $\varepsilon_{1}$ and $\varepsilon_{2}$ could both happen on the cubic LAO unit cells and form the resulting incommensurate stacking sequence in different directions. 


\section{References}

1. Kolb, U., Gorelik, T. \& Otten, M.T. Towards automated diffraction tomography. Part II—Cell parameter determination. Ultramicroscopy 108, 763-772 (2008).

2. Palatinus, L. PETS (program for analysis of electron diffraction data). Institute of Physics of the AS CR, Prague, Czechia (2011).

3. Petricek, V., Dusek, M., \& Palatinus, L. Jana2006. Structure Determination Software Programs. Institute of Physics, Praha, Czech Republic.

4. Boullay, P., Palatinus, L. \& Barrier, N. Precession Electron Diffraction Tomography for Solving Complex Modulated Structures: the Case of $\mathrm{Bi}_{5} \mathrm{Nb}_{3} \mathrm{O}_{15}$. Inorg. Chem. 52, 6127-6135 (2013).

5. Wang, Y., Lv, J., Zhu, L. \& Ma, Y. CALYPSO: A method for crystal structure prediction. Comput. Phys. Commun. 183, 2063-2070 (2012).

6. Heyd, J., Scuseria, G.E. \& Ernzerhof, M. Hybrid functionals based on a screened Coulomb potential. J. Chem. Phys. 118, 8207-8215 (2003).

7. Blöchl, P.E. Projector augmented-wave method. Phys. Rev. B 50, 17953-17979 (1994).

8. Kresse, G. \& Joubert, D. From ultrasoft pseudopotentials to the projector augmented-wave method. Phys. Rev. B 59, 1758-1775 (1999).

9. Kresse, G. \& Furthmüller, J. Efficiency of ab-initio total energy calculations for metals and semiconductors using a plane-wave basis set. Comp. Mater. Sci. 6, 1550 (1996).

10. Kresse, G. \& Furthmüller, J. Efficient iterative schemes for $a b$ initio total-energy calculations using a plane-wave basis set. Phys. Rev. B 54, 11169-11186 (1996). 